cytes themselves, which, as they become more numerous, tend to migrate to the surface of the egg and finally form into one or more layers. Each nucleus apparently acquires a local area or zone of protoplasm which ultimately becomes marked off from adjacent areas as more or less of a definite cell. The pigment of the egg accumulates around the boundaries of the more superficial areas, which thus appear to be sharply delimited, as seen in sections under the microscope. Although the internal mass of yolk contains numerous nuclei, frequently undergoing amitotic division, the central mass of the eggs remains in a syncitial condition for considerable time.

I am inclined to believe that, in some cases at least, the female pronucleus of the egg takes no part in this cell proliferation, because I have been able to find in sections in several instances a comparatively large clear protoplasmic zone, variously placed in the egg, which, although invaded more or less on all sides by nuclei, itself remains undivided, and in it is visible what appears to be a degenerating nuclear-like structure, presumably the remainder of the female pronucleus.

Many eggs show no development, but this is not to be wondered at, since doubtless a number of them, although pricked, received no corpuscles from the orifice of the capillary tube. Other eggs, presumably not fully ready for fertilization, did not develop; although the corpuscles apparently proliferated extensively, they later ran together to form giant cells and frequently seemed to become phagocytic in nature. In still other cases, what seemed to be phagocytosis was visible on one side of the egg, while on the other side the nuclei appeared to be ranging up into a defnite cellular layer. A detailed description of the experiments is in preparation.

The University of CincinNati, Midhael F. Guyer May 13, 1907

TRANSMISSION INHERITANCE DISTINCT FROM EXPRESSION INHERITANCE ${ }^{1}$

Conjugations of sex-cells of higher plants and animals have two results, an intermediate

${ }^{1}$ Read before the Botanical Society of Washington, April 13, 1907. and a final product. The intermediate product of conjugation is a new organism, the final product a new equipment of sex-cells. The new organism is built up by vegetative subdivisions of the conjugating pair of sexcells. The conjugation is not completed until the new generation of sex-cells is to be formed. Fertilization is the beginning of the process of conjugation, which may not conclude for months or years after fertilization has taken place. The organism which is built up during conjugation may be called a conjugate organism, or conjugate. It belongs to the same generation as the sex-cells which initiate the conjugation. The next generation may be called perjugate, since it has passed through the conjugation of the preceding generation and represents its completed results.

When sex-cells of diverse parentage are associated in conjugation the organisms they build up (conjugates) may be like one parent, or like both parents, or intermediate between the two parents, or different from either parent. The same latitude of alternatives of expression is found in the perjugate generation. The crossing of two varieties of pink-eyed mice yields black-eyed conjugates. Two varieties of smooth-seeded cottons gave smooth-seeded conjugates, but woolly-seeded perjugates. Such instances prove that the expression-tendency of a gamete can be altered by association with another gamete of diverse parentage. Either the conjugate generation or the perjugate generation, or both, may show characters which neither of the parent gametes would have brought into expression if it had secured a partner of its own kind. There is no corresponding proof that transmission inheritance is altered by such associations. The reappearance of such characters as the black eyes and the woolly seeds, which have been abeyant through many generations, shows that failure of expression does not prove failure of transmission.

Transmission inheritance may be thought of as the dial of a compass which carries many character-directions, though the needle of expression points to only one. This expressionpolarity is called dominance in conjugate organisms and potency in gametes. Nobody 
denies the transmission of unexpressed characters through conjugate organisms, but in dealing with gametes the distinction between transmission and expression has continued to be overlooked; otherwise the Mendelian hypothesis of pure germ-cells could not have attained its wide popularity.

Mendelism and other forms of polarized expression inheritance yield us no intimation whatever regarding the nature and mechanism of transmission inheritance. If transmission could be conceived as a matter of localized character-unit particles we should be justified in thinking of all germ-cells as containing full sets, and not variously mangled fractions of the ancestral equipments. Alternative inheritance of divergent characters means reciprocal expression-polarities. It has yet to be shown that there is any such phenomenon as alternative transmission inheritance, brought about by the segregation of the parental character-units in different germ-cells. Incompatibility sufficient to cause germinal segregation should preserve the original association of the characters, but no such tendency has appeared in Mendelian crosses. When there are several divergent characters they are always expressed in many different combinations, as though to show that the scale of transmission remains complete, no matter how narrowly the needle of expression may sometimes be directed.

O. F. Coor

\section{A NEW METHOD BY WHICH SPONGES MAY BE ARTIFICIALLY REARED ${ }^{3}$}

I HAVE found in the course of an investigation carried on for the Bureau of Fisheries that silicious sponges when kept in confinement under proper conditions degenerate, giving rise to small masses of undifferentiated tissue which in their turn are able to grow and differentiate into perfect sponges. The investigation has been prosecuted during the past three summers at the Beaufort Laboratory. While the degeneration with the formation of the indifferent masses has been ob-

${ }^{2}$ Published with the permission of Hon. Geo. M. Bowers, U. S. Commissioner of Fisheries. served in several species, it is only in ono species, a Stylotella, that the process as a whole has been worked out.

This sponge, which is exceedingly abundant in Beaufort Harbor, is a fleshy monactinellid commonly reaching a thickness and height of 10-12 cm. Conical processes with terminal oscula project upwards from the lower body. With this species, which is a light-loving form, I have obtained the best results when outside aquaria, either concrete aquaria or tubs, were used. The method of treatment is briefly this : Into a tub about $60 \mathrm{~cm}$. by $30 \mathrm{~cm}$. and covered with glass, a half dozen sponges, freed as far as possible from live oysters and crabs, are put. They are raised from the bottom on bricks. The tub is emptied, filled and flushed for some minutes three times in every twentyfour hours. Direct rays of the sun should be avoided. Tubs answer as well as concrete aquaria, and have the advantage of being movable.

In a day or two the oscula of the sponge disappear, and the surface begins to acquire a peculiar smooth, dense and uniform appearance. Microscopic study reveals the fact that not only the oscula, but the pores also, for the most part, close, and the canal system becomes interrupted and in some degree suppressed. The mesenchyme is more uniform, and is denser than in the normal sponge, owing in part at least to the disappearance of the extensive collenchymatous (very watery mesenchyme) tracts of the latter.

The whole sponge may pass into this state and remain without great change for weeks. During this period it shrinks greatly in size, in a given case to one quarter the original bulk. The arrangement of the skeletal spicules becomes much simplified. With the shrinkage in size the sponge becomes more solid, $i$. e., more of the canal space is suppressed. Some flagellated chambers persist and there are a few small scattered apertures on the surface. The bulk of the chambers disappear as such, the collar-cells transforming into simple polyhedral masses which become scattered singly or in groups in the general mesenchyme. The mesenchyme is a syncytium composed of well-marked cells that are 